\title{
AGROBIODIVERSIDAD AMAZÓNICA Y ESTRATEGIAS DE DESARROLLO LOCAL SOSTENIBLE ${ }^{1}$
}

\section{AMAZON AGROBIODIVERSITY AND LOCAL SUSTAINABLE DEVELOPMENT STRATEGIES}

\author{
Ruth Irene Arias-Gutiérrez ${ }^{*}$ \\ Roberto González Sousa** \\ Angelina Herrera Sorzano*** \\ Manuel Lázaro Pérez Quintana****
}

RESUMEN

\begin{abstract}
En este artículo se analiza el vínculo entre agrobiodiversidad y formación del capital humano en el desarrollo de comunidades rurales de la Amazonía ecuatoriana. Se cuantifican recursos del bosque y agropecuarios, satisfacción de necesidades alimentarias e impacto de instituciones académicas en comunidades del valle del río Anzu. El mejoramiento de cultivos asociados y el bio-conocimiento son las bases para establecer estrategias que favorezcan el cambio de la matriz productiva en los planes de desarrollo.
\end{abstract}

PALABRAS CLAVE: ECUADOR * RECURSOS RENOVABLES * COMUNIDADES RURALES *
DESARROLLO SOSTENIBLE * EDUCACIÓN AMBIENTAL

ABSTRACT

In this article, is analyzed the link between agricultural biodiversity and human capital formation in rural communities development in the Ecuadorian Amazon. Forest and

1 Se agradece a la Secretaría de Educación Superior, Ciencia, Tecnología e Innovación del Ecuador (SENESCYT) y a la Universidad Estatal Amazónica (UEA), de Puyo Ecuador, por el apoyo financiero al desarrollo de la presente investigación, así como a la Universidad de la Habana, Cuba por el apoyo académico brindado.

* Universidad Estatal Amazónica, Ecuador.

rarias@uea.edu.ec

** Facultad de Geografía, Universidad de La Habana, Cuba. rgsousa@geo.uh.cu

*** Facultad de Geografía, Universidad de La Habana, Cuba. aherrera@geo.uh.cu

**** Universidad Estatal Amazónica, Ecuador. mperez@uea.edu.ec 
agricultural resources, alimentary needs and academic institutions impact in Anzu River Valley communities are quantified. Improving cropping and bio-knowledge are the basis for strategies that change the productive matrix in development plans.

KEYWORDS: ECUADOR * RENEWABLES RESOURCES * RURAL COMMUNITIES * SUSTAINABLE DEVELOPMENT * ENVIROMENTAL EDUCATION

\section{INTRODUCCIÓN}

Las aspiraciones de desarrollo sustentable buscan coherencia entre las esferas disciplinares social, económica y ambiental, en una cultura de equidad y paz (Cantú 2012; Córdoba 1996); las experiencias de desarrollo relacionadas con la revolución verde, establecidas en todo el mundo desde la década del 50 del siglo xx, provocaron desigualdad social, dependencia y subdesarrollo, con profunda degradación medioambiental, social y cultural, difíciles de revertir (Escobar 2002). Todavía en el año 2012, el $20 \%$ de la población ecuatoriana más pobre tenía el 4,1\% del ingreso del país, mientras el $20 \%$ de la población más rica mantenía el $52,5 \%$ (Centro de Derechos Económicos y Sociales-cDes 2013).

Las provincias amazónicas, con mayor población rural, figuran entre las más pobres en el Ecuador (Instituto Nacional de Estadísticas y Censos-inec 2010); en estas se desarrolló un proceso de colonización espontánea, especialmente por pobladores pobres y sin capital, a diferencia de la colonización de la Amazonía en países como Brasil, facilitada por infraestructura y asistencia estatal (Gudynas 2004; Bilsborrow, Barbieri y Pan 2004; Zambrana 2011) o la Amazonía boliviana por grandes hacendados con acumulación patrimonial capitalista (García 2012).

La colonización en la Amazonía ecuatoriana afectó a los pueblos indígenas, llamados naciones originarias (Bartolomé 2010; Vázquez 2012). Una de estas, la nación Kichwa o Quichua, ubicada en cuatro provincias amazónicas, en el norte y centro de la región, desde las estribaciones orientales de la Cordillera Real de los Andes al Oeste, hasta la frontera con Perú al Este, logró legalizar una parte de su territorio en la selva en 1992. En la zona de colonización en las estribaciones de la cordillera, quedaron comunidades sin legalizar, con sus formas tradicionales biodiversas de uso del territorio (Philip 2011, Pérez y Zárate 2011).

Prates (2009) refiere que la porción occidental de la Amazonía - donde está Ecuador- es una de las áreas más biodiversas del planeta y el hogar de varios pueblos indígenas, con porciones intactas de bosque muy húmedo tropical y una alta probabilidad de estabilizar las condiciones climáticas frente a los problemas del calentamiento global. La conservación de la biodiversidad y el manejo sustentable de los ecosistemas en los niveles global, nacional $y$ local son elementos clave en las políticas $y$ estrategias de reducción de la pobreza para el $70 \%$ de los pobres del mundo que viven en áreas rurales y dependen directamente de la biodiversidad para su supervivencia $y$ bienestar (Álvarez y Shany 2012, Gaona 2013).

Las comunidades indígenas no tienen un sentido de acumulación capitalista, mantienen su sentido de generosidad, reciprocidad y bien común, sus territorios en la zona de colonización conservan bosques. Hay una relación de continuidad entre el mundo humano, el biofísico y el natural (Escobar 2002). Las áreas de ecoturismo, investigación científica, agroforestería, extracción y procesamiento de productos del bosque, se consideran desde hace décadas como alternativas de ingresos para los pobladores amazónicos, en una región donde los ingresos de la agricultura son en promedio, los más bajos a nivel nacional, los esfuerzos por encontrar alternativas de ingresos para los campesinos son necesarios y las posibilidades de desarrollo dependen de educación, infraestructura e inversión (Vasco et ál. 2013).

Por otra parte, se requiere la producción del conocimiento científico que considere el ámbito étnico propio de las comunidades, sus posibilidades y sus recursos en el territorio, 
en términos de sostenibilidad, porque los modelos de desarrollo asignados a la región amazónica no han significado beneficio para sus pobladores, hay cambios en el uso del territorio $y$ un déficit en el aprovechamiento de los recursos de la agrobiodiversidad amazónica.

Por las necesidades actuales de las comunidades indígenas en vestimenta, estudio, salud y transporte, entre otras, así como por las propias reflexiones sobre sus perspectivas de futuro, es necesario investigar opciones de desarrollo local sostenibles que no degraden la naturaleza, considerada como la Pachamama o la madre tierra, gestora de vida. Esas consideraciones sitúan a las opciones agropecuarias con valor agregado $y$ de formación del capital humano como nuevas perspectivas de desarrollo.
El objetivo del presente trabajo fue analizar el vínculo entre el aprovechamiento de recursos de la agrobiodiversidad amazónica, la formación del capital humano y su posible impacto en el desarrollo de comunidades rurales en el territorio Kichwa colonizado en la Amazonía ecuatoriana, así como proponer las bases para una estrategia de desarrollo local sostenible para esos entornos rurales.

\section{LOCALIZACIÓN Y CARACTERÍSTICAS GEOGRÁFICAS}

La figura 1 y el cuadro 1 muestran la localización de las seis comunidades en estudio, desde Tzawata al Noreste; hasta Veinticuatro de Mayo al Suroeste, ubicadas en los niveles bajo, medio y alto del valle del río Anzu. 


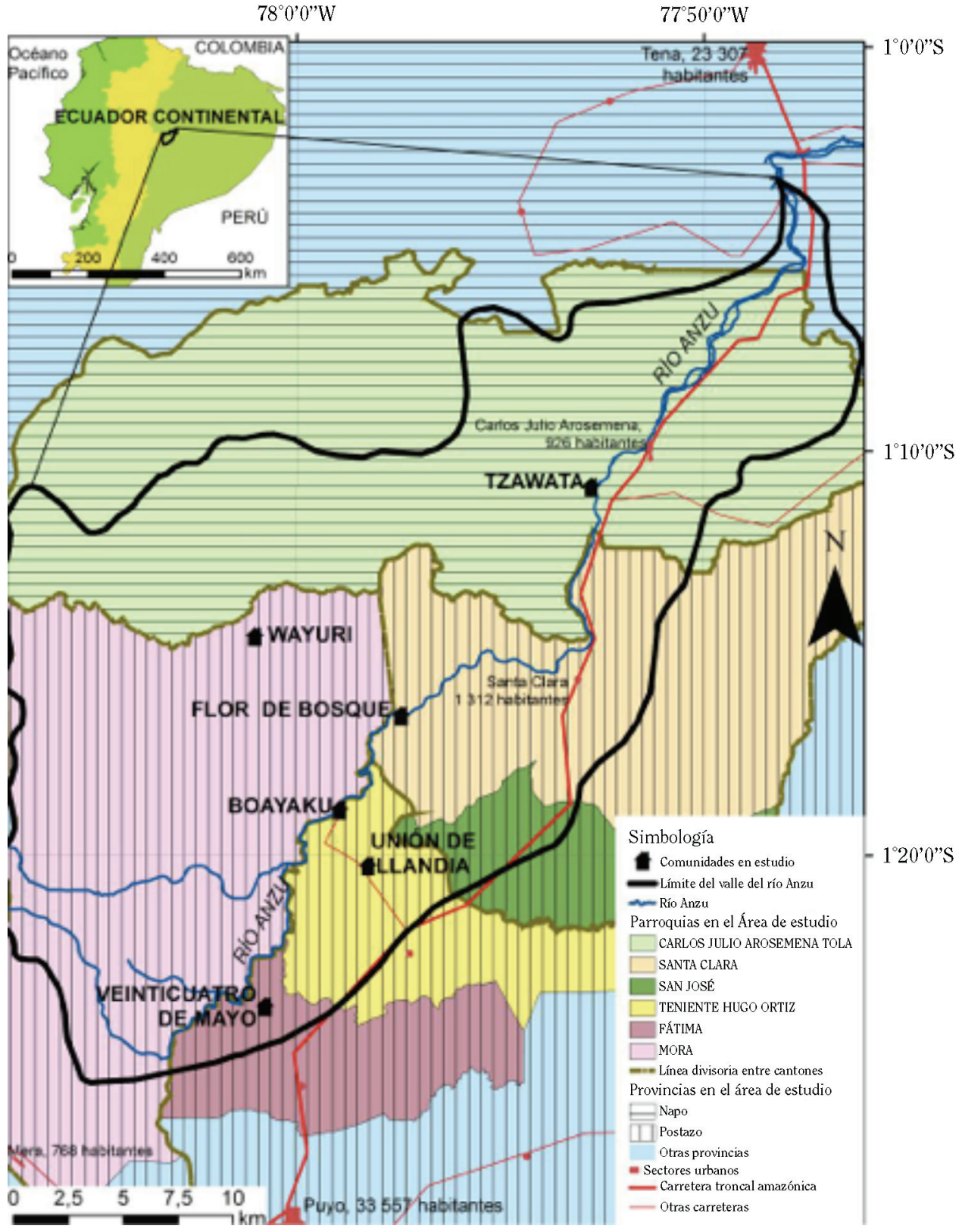

FIGURA 1

LOCALIZACIÓN DEL

ÁREA DE ESTUDIO

Fuente: Elaboración propia con base en los datos de campo de la investigación e información oficial disponible en www.sni.gob.ec 
CUADRO 1

LOCALIZACIÓN DE LAS COMUNIDADES EN ESTUDIO

\begin{tabular}{cccccccc}
\hline COMUNIDAD & $Z^{*}$ & ALTITUD & LATITUD & LONGITUD & PARROQUIA & CANTÓN & PROVINCIA \\
\hline Tzawata & 18 & 508 & 9869264 & 179523 & $\begin{array}{c}\text { Carlos Julio } \\
\text { Arosemena }\end{array}$ & $\begin{array}{c}\text { Carlos Julio } \\
\text { Arosemena }\end{array}$ & Napo \\
\hline Wayuri & 17 & 1200 & 9862500 & 831306 & Mera & Mera & Pastaza \\
\hline Flor de Bosque & 18 & 752 & 9858789 & 170876 & Santa Clara & Santa Clara & Pastaza \\
\hline Boayaku & 18 & 808 & 9854478 & 168082 & $\begin{array}{c}\text { Teniente Hugo } \\
\text { Ortiz }\end{array}$ & Pastaza & Pastaza \\
\hline Unión de Llandia & 18 & 1099 & 9851931 & 169383 & $\begin{array}{c}\text { Teniente Hugo } \\
\text { Ortiz }\end{array}$ & Pastaza & Pastaza \\
\hline $\begin{array}{c}\text { Veinticuatro de } \\
\text { Mayo }\end{array}$ & 17 & 994 & 9845509 & 832473 & Fátima & Pastaza & Pastaza \\
\hline
\end{tabular}

Fuente: Elaboración propia con base en los datos de la investigación de campo.

Nota*: Zona UTM 17 Sur, WGS 1984.

Las comunidades en estudio se localizan en el territorio colonizado de la nación originaria Kichwa amazónica ecuatoriana, se asentaron desde finales del siglo xIx y provienen de la provincia de Napo. Se exceptúa Unión de Llandia, con más población de campesinos migrantes de la región interandina o sierra, producto del proceso de colonización de la década de 1960. Se ubican desde los 508 m s. n. m. hasta los $1200 \mathrm{~m} \mathrm{~s}$. n. m. en las estribaciones orientales de los Andes centrales, al sur de la zona sub andina identificada como levantamiento Napo; sobre suelos aluviales hidromórficos de vocación forestal y origen volcánico, con fines de conservación; suelos formados por los conos de deyección en el piedemonte amazónico, como consecuencia de la fusión de los casquetes glaciares plio-cuaternarios y de la actividad volcánica y sísmica (Senplades e Instituto Geográfico Militar-IGM 2010).

La pluviosidad supera los $6000 \mathrm{~mm}$ anuales; la temperatura promedio se establece entre 20 y $24{ }^{\circ} \mathrm{C}$; el clima es tropical húmedo; la topografía corresponde a terrenos quebrados y colinados, con pendientes que varían desde terrenos relativamente planos en las zonas más bajas, hasta inclinaciones de $70^{\circ}$ o más, en las altitudes mayores. Prevalecen paisajes de los trópicos permanentemente húmedos con bosque tropical (González y Salinas 2010). La zona de vida corresponde a bosque pluvial premontano (Cañadas y Cruz 1983); la formación vegetal es bosque siempre verde piemontano (Sierra 1999).

\section{METODOLOGÍA}

Se seleccionaron las comunidades a estudiar con base en su vulnerabilidad, por encontrarse inmersas en un sector de colonización que extrae recursos y destruye la base material de existencia de las comunidades.

Se diseñó el acercamiento a la realidad de las comunidades, a través de eventos de discusión y sus organizaciones, realizados entre julio de 2012 y julio de 2014. Se usó observación participante, análisis documental y recuperación de la historia oral, métodos cualitativos apropiados a la realidad de estudio en proceso (Blanke y Walzer 2013; Montero 2011). Se aplicaron encuestas a los pobladores y directivos de las seis comunidades $y$ a los jefes de los 64 hogares dispersos en un área que cubre cinco parroquias rurales. Se tabularon los datos 
de las encuestas y su análisis se presenta como resultado en el presente trabajo.

Entre los indicadores medidos se utilizaron el aprovechamiento de recursos de la agrobiodiversidad amazónica; grado en que el territorio local satisface las necesidades alimentarias de cada familia; cambios de uso en los sistemas agrícolas; educación, servicios y condiciones de las viviendas en las comunidades.

\section{RESULTADOS Y DISCUSIÓN}

APROVECHAMIENTO DE LA AGROBIODIVERSIDAD

El cuadro 2 presenta hasta 107 especies cultivadas en sistemas de chacras en las comunidades Lorocachi, Nina Amarun, Victoria y Yana Yacu (Vacacela e Instituto Quichua de Biotecnología Sacha Supai 2013), referenciales en la selva prístina, lejos del sector colonizado donde se asientan las comunidades en estudio.

\section{CUADRO 2}

NÚMERO DE ESPECIES CULTIVADAS EN SISTEMAS DE CHACRAS EN COMUNIDADES KICHWA

\begin{tabular}{lccccccc}
\hline COMUNIDAD & ALIMENTO & MEDICINA & RITUAL & SABOR & COSMÉTICA & TÓXICA & TOTAL \\
\hline Lorocachi & 63 & 15 & 19 & 6 & 3 & 1 & 107 \\
Nina Amarun & 44 & 8 & 7 & 4 & 3 & 1 & 67 \\
Victoria & 51 & 5 & 14 & 4 & 2 & 1 & 77 \\
Yana Yacu & 45 & 5 & 7 & 3 & 1 & 1 & 62 \\
\hline
\end{tabular}

Fuente: Elaboración propia con datos de Vacacela-IQBSs, Puyo 2013.

En esas comunidades de referencia se observa que emplean un elevado número de especies como alimento directamente y un número menor para medicina, saborizantes y cosméticos. Se destacan algunas especies empleadas como ritual y solo una especie tóxica. Mientras que en el sector colonizado, las chacras no superan las 30 especies asociadas.

El ciclo chacra-ushun-purun es un sistema agrobiodiverso característico y generalizado en comunidades Kichwa y en otros pueblos indígenas; posee cultivos asociados que satisfacen las necesidades alimentarias anuales de la familia, especialmente con yuca, ají, calabaza, papaya, plátanos, piñas y otras especies alimenticias; se instala en bosque primario o secundario para aprovechar la fertilidad orgánica del suelo, se cultiva varios años; luego del primer año o las primeras cosechas, toma el nombre de ushun, consistente en la resiembra de yuca, otros tubérculos y el incremento de plátanos, palmas útiles $y$ árboles frutales. Mientras se cosecha yuca y plátanos este sistema se denomina ushun, después, se transforma en purun, estado de regeneración natural en el que se mantienen especies frutales comestibles, medicinales, forestales, en configuraciones boscosas algo similares a la de los bosques primarios (Landázuri 2013). El ciclo es testimonio del trabajo de la familia y la comunidad, huella en el territorio para reconocimiento de límites y conservación de la biodiversidad con especies que pueden ser útiles en la obtención de biopreparados con fines médicos, medicinales y alimenticios.

El manejo cíclico de la chacra-ushunpurun garantiza la conservación de suelo, bosque y biodiversidad; implica prácticas de las comunidades locales con un enfoque desde la cultura y del control del territorio propio (Escobar 2002; Martínez 2012). Las comunidades Kichwa tienen gran conocimiento del uso de los recursos de sus ecosistemas como materias primas para cocinar, alimentación humana $y$ animal, así como para la construcción de viviendas 
(cuadro 3), pero no tienen una explotación ordenada $y$ eficiente en beneficio de las comunidades, donde se observe aplicación de la ciencia y la tecnología para añadir valor al recurso; no tienen relaciones con procesos agroindustriales que permitan la obtención de productos elaborados con valor agregado, lo que significa una debilidad que pudiera mejorarse con el vínculo a proyectos científicos ejecutados con las universidades del territorio (Martínez 2012).

CUADRO 3

USOS POR ESPECIES EXISTENTES EN EL SISTEMA TERRITORIAL INDÍGENA KICHWA

\begin{tabular}{|c|c|c|c|c|}
\hline $\begin{array}{c}\text { CATEGORÍA DE USO } \\
\text { DE RECURSO RENOVABLE NATIVO }\end{array}$ & $\begin{array}{c}\text { FLORA } \\
\text { (DE } 366 \\
\text { ESPECIES) }\end{array}$ & $\begin{array}{c}\text { MAMÍFEROS } \\
\text { (DE } 28 \\
\text { ESPECIES) }\end{array}$ & $\begin{array}{c}\text { AVES } \\
\text { (DE } 51 \\
\text { ESPECIES) }\end{array}$ & $\begin{array}{c}\text { PECES } \\
\text { (DE } 141 \\
\text { ESPECIES) }\end{array}$ \\
\hline Leña & 266 & - & - & - \\
\hline Alimento mamíferos & 202 & - & - & - \\
\hline Alimento aves & 171 & - & - & - \\
\hline Alimento Humano & 70 & 30 & 25 & 133 \\
\hline Medicina & 28 & 4 & 1 & 2 \\
\hline Maderable & 62 & - & - & - \\
\hline Construcción & 69 & - & - & - \\
\hline Alimento peces & 5 & - & - & - \\
\hline Artesanal & 9 & 21 & 23 & 1 \\
\hline Mitológico & 2 & 3 & 1 & 9 \\
\hline Ictiológico & 2 & - & - & - \\
\hline Mascota & - & 11 & 2 & - \\
\hline Cebo/ carnada & - & 1 & 2 & - \\
\hline TOTAL USOS & 886 usos & 70 usos & 54 usos & 145 usos \\
\hline
\end{tabular}

Fuente: Elaboración propia con base en los datos de Vacacela-IQBSS 2013.

El sistema indígena en el territorio amazónico permanece relacionado con el sistema ambiental, proporciona fuentes de subsistencia $y$ beneficia especies de la biodiversidad que usan y aprovechan; los Kichwa reconocen tenencia tradicional comunitaria del territorio para la comunidad o muntun (familia ampliada y sistema de relaciones de parentesco y alianzas). La comunidad reconoce para cada ayllu (familia lineal) un espacio de uso en el cual establecen sus casas, chacras (cultivos asociados) y purinas (cotos de caza y pesca en los que permanecen parte del año). El producto del trabajo es familiar, si hay tierra comunitaria disponible se asigna a los socios que necesiten y soliciten (jóvenes que forman familia, familiares o relacionados que regresan o ingresan a la comunidad).

En el cuadro 4 se presentan los recursos renovables más aprovechados por las comunidades en estudio. 


\section{CUADRO 4 \\ RECURSOS RENOVABLES DE APROVECHAMIENTO DEL BOSQUE \\ Y DESTINO DE USO EXPRESADO \\ 2012}

\begin{tabular}{|c|c|c|c|c|}
\hline \multirow[t]{2}{*}{ COMUNIDAD } & \multicolumn{2}{|c|}{ RECURSOS RENOVABLES DEL BOSQUE } & \multirow{2}{*}{$\begin{array}{l}\text { PARA USO } \\
\text { DOMÉSTICO }\end{array}$} & \multirow{2}{*}{$\begin{array}{r}\text { PARA } \\
\text { VENTA }\end{array}$} \\
\hline & MADERABLES & NO MADERABLES & & \\
\hline Tzawata & $\begin{array}{l}\text {-Maderas finas: chuncho, } \\
\text { ahuano, cedro, laurel. }\end{array}$ & $\begin{array}{l}\text {-Animales comestibles: guanta. } \\
\text {-Frutos comestibles: hungurahua. } \\
\text {-Productos medicinales: guayusa, uña de } \\
\text { gato, cortezas, hojas. } \\
\text {-Productos para artesanía: fibras. }\end{array}$ & 70 & 30 \\
\hline Wayuri & -Madera suave: leña. & $\begin{array}{l}\text {-Animales comestibles: no declaran. } \\
\text {-Frutos comestibles: chonta. } \\
\text {-Productos medicinales: chugchuhuazo, } \\
\text { guayusa. } \\
\text {-Productos para artesanía: no declaran. }\end{array}$ & 100 & 0 \\
\hline $\begin{array}{l}\text { Flor de } \\
\text { Bosque }\end{array}$ & $\begin{array}{l}\text {-Maderas finas: canelo, } \\
\text { laurel. } \\
\text {-Madera suave: pihue, } \\
\text { leña. }\end{array}$ & $\begin{array}{l}\text {-Animales comestibles: armadillo, } \\
\text { guatusa, guanta. } \\
\text {-Frutos comestibles: cúrcuma, ishpingo. } \\
\text {-Productos medicinales: uña de gato, } \\
\text { sangre de drago. } \\
\text {-Productos para artesanía: fibras, } \\
\text { chambira, pita. }\end{array}$ & 70 & 30 \\
\hline Boayaku & $\begin{array}{l}\text {-Maderas finas: canelo, } \\
\text { chuncho, laurel. } \\
\text {-Madera suave: pihue. }\end{array}$ & $\begin{array}{l}\text {-Animales comestibles: sajino. } \\
\text {-Frutos comestibles: no declaran. } \\
\text {-Productos medicinales: mushukhuan. } \\
\text {-Productos para artesanía: fibras, } \\
\text { chambira. }\end{array}$ & 7 & 93 \\
\hline $\begin{array}{l}\text { Unión de } \\
\text { Llandia }\end{array}$ & $\begin{array}{l}\text {-Madera dura: canelo. } \\
\text { Madera suave: pihue. }\end{array}$ & $\begin{array}{l}\text {-Animales comestibles: no declaran. } \\
\text {-Frutos comestibles: no declaran. } \\
\text {-Productos medicinales: no declaran. } \\
\text {-Productos para artesanía: no declaran. }\end{array}$ & 21 & 79 \\
\hline $\begin{array}{l}\text { Veinticuatro } \\
\text { de Mayo }\end{array}$ & $\begin{array}{l}\text {-Madera dura: canelo. } \\
\text {-Madera suave: pihue, } \\
\text { leña. }\end{array}$ & $\begin{array}{l}\text {-Animales comestibles: guatusas, monos. } \\
\text {-Frutos comestibles: palmito. } \\
\text {-Productos medicinales: no declaran. } \\
\text {-Productos para artesanía: no declaran. }\end{array}$ & 42 & 58 \\
\hline
\end{tabular}

Fuente: Elaboración propia a partir de las encuestas a las familias. 
Se observa que, excepto Wayuri, que no tiene carretera cerca, todas las comunidades extrajeron maderas finas o duras para construcción y muebles. Unión de Llandia, con mayor porcentaje de población mestiza y cerca de la carretera, no reportó uso de plantas medicinales ni animales silvestres comestibles. En las comunidades colonizadas, Unión de Llandia y Veinticuatro de Mayo, se extrajo menor variedad de productos forestales y no forestales. Boayaku, comunidad colonizada, con bastante población Kichwa, se ubica cerca del bosque, al final de una carretera de la colonización, constituye una frontera entre la colonización y la selva, se destaca el porcentaje de recursos para la venta. Las comunidades Tzawata, Wayuri y Flor de Bosque, de población 100\% Kichwa declaran mayor uso doméstico, mientras las tres restantes tienen un mayor destino para venta de recursos.

Entre los problemas ambientales más críticos de Ecuador, que devienen en obstácu- los al desarrollo, se destacan, entre otros, la irracional explotación no sustentable de los recursos naturales no renovables que ha provocado contaminación, riesgos y amenazas socio económicas y culturales a los pobladores de las zonas amazónicas afectadas (SENPLADEs e IGM 2010), que sin embargo mantienen su riqueza natural, biológica, cultural y conocimientos. Las comunidades tienen gran dependencia del medio amazónico, es importante analizar sus relaciones.

GRADO EN QUE EL TERRITORIO LOCAL SATISFACE LAS NECESIDADES ALIMENTARIAS DE CADA FAMILIA

En el cuadro 5 se indica en porcentaje, la percepción de dependencia de la familia respecto al sistema local de productos, es decir, respecto a los recursos obtenidos del medio local, el bosque, animales, pesca y los cultivados por la familia.

CUADRO 5

PORCENTAJE DE DEPENDENCIA FAMILIAR RESPECTO A LOS RECURSOS LOCALES Y DEL BOSQUE O SELVA

2012

\begin{tabular}{|c|c|c|c|c|c|c|}
\hline $\begin{array}{l}\text { APROVECHAMIENTO } \\
\text { RECURSOS LOCALES }\end{array}$ & TZAWATA & WAYURI & $\begin{array}{l}\text { FLOR DE } \\
\text { BOSQUE }\end{array}$ & BOAYAKU & $\begin{array}{l}\text { UNIÓN DE } \\
\text { LLANDIA }\end{array}$ & $\begin{array}{l}\text { VEINTICUATRO } \\
\text { DE MAYO }\end{array}$ \\
\hline Declaran uso & 100 & 67 & 89 & 100 & 90 & 100 \\
\hline $\begin{array}{l}\text { Familias Kichwa que } \\
\text { declaran uso }\end{array}$ & 100 & 67 & 89 & 100 & 100 & 100 \\
\hline $\begin{array}{l}\text { Familias mestizas que } \\
\text { declaran uso }\end{array}$ & 0 & 0 & 0 & 100 & 87,5 & 100 \\
\hline $\begin{array}{l}\text { Total provecho familias } \\
\text { Kichwa }\end{array}$ & 100 & 100 & 100 & 90 & 22 & 60 \\
\hline $\begin{array}{l}\text { Total provecho familias } \\
\text { mestizas }\end{array}$ & 0 & 0 & 0 & 10 & 78 & 40 \\
\hline Etnicidad de referencia & Kichwa 100 & Kichwa 100 & Kichwa 100 & $\begin{array}{c}\text { Kichwa } \\
85 \\
\text { Mestiza } \\
15\end{array}$ & $\begin{array}{c}\text { Kichwa } \\
20 \\
\text { Mestiza } \\
80\end{array}$ & $\begin{array}{c}\text { Kichwa } \\
50 \\
\text { Mestiza } \\
50\end{array}$ \\
\hline Informantes & 12 & 6 & 9 & 13 & 20 & 4 \\
\hline
\end{tabular}

Fuente: Elaboración propia a partir de las encuestas a las familias. 
Se observa que al menos el $67 \%$ de todas las familias declara uso o aprovechamiento de los recursos locales comprendidos en el bosque o selva, ríos y la finca que provee alimentos cultivados o animales de crianza. Los comuneros de Wayuri permanecen en Santa Clara por la escuela de los niños, en general tienen empleo, eso explica que no declaren mayor porcentaje de aprovechamiento. También en Flor de Bosque aparece menor aprovechamiento por declarantes con empleo fuera de la comunidad. La diferencia entre el provecho que logran las familias mestizas y Kichwa está en relación con su peso poblacional: de la sumatoria de aprovechamientos declarados se extrae el reportado por familias Kichwa y el declarado por familias mestizas. La búsqueda de empleos en los poblados previene los cambios en las familias rurales.

\section{CAMBIOS DE USO EN LOS SISTEMAS AGRÍCOLAS}

Además de la yuca y el plátano, en las chacras también aparecen algunos cultivos comerciales como la naranjilla (Solanum quitoensis), baya globosa apreciada localmente pero que requiere herbicida químico, la caña de azúcar (Saccharum sp.) para fruta y aguardiente, en forma de monocultivo, con gran cantidad de fertilizante; la papachina o malanga (Colocasia sculenta), tubérculo que se procesa en la Parroquia Teniente Hugo Ortiz; el cacao fino de aroma (Theobroma so.). Los cultivos comerciales en función de la alta pluviosidad, requieren asistencia política, técnica $y$ administrativa. Excepto esos cultivos, los demás son cultivos asociados entre sí y con más especies, en formas de organización agroecológica que asignan las comunidades y que no se han estudiado suficiente.

Las chacras alimentan a las familias todo el año, tradicionalmente significan soberanía alimentaria. El aparecimiento de cultivos comerciales en las chacras y el aumento de la extensión de cada una de estas - que normalmente es menor a una hectárea- indica que las comunidades se están orientando a cultivar más productos destinados al mercado que los de subsistencia, aunque no sea en la totalidad de familias. En comunidades indígenas amazónicas, la producción para el mercado es reciente por lo que se necesita asesoramiento y desarrollo de proyectos que bien pueden provenir de instituciones académicas de nivel superior.

\section{EDUCACIÓN, SERVICIOS Y CONDICIONES DE LAS VIVIENDAS}

El cuadro 6 muestra el estado de la educación, servicios y condiciones de las viviendas en las comunidades en estudio.

CUADRO 6

INDICADORES DE EDUCACIÓN, SALUD, SERVICIOS Y CONDICIONES DE LAS VIVIENDAS EXPRESADOS EN PORCENTAJE

\begin{tabular}{lcccccc}
\hline CONDICIÓN O SERVICIO & TZAWATA & WAYURI & $\begin{array}{c}\text { FLOR DE } \\
\text { BOSQUE }\end{array}$ & BOAYAKU & $\begin{array}{c}\text { UNIÓN DE } \\
\text { LLANDIA }\end{array}$ & $\begin{array}{c}\text { VEINTICUATRO } \\
\text { DE MAYO }\end{array}$ \\
\hline Analfabetos & 0 & 0 & 0 & 8 & 20 & 25 \\
\hline $\begin{array}{l}\text { Alfabetizados y/o primaria } \\
\text { incompleta }\end{array}$ & 33 & 0 & 11 & 0 & 5 & 25 \\
\hline Ed. primaria completa & 59 & 34 & 23 & 38 & 55 & 50 \\
\hline Secundaria completa & 8 & 33 & 11 & 15 & 5 & 0 \\
\hline Bachillerato & 0 & 0 & 11 & 0 & 0 & 0 \\
\hline Superior completa & 0 & 0 & 11 & 8 & 0 & 0 \\
\hline Con red de agua & 0 & 67 & 22 & 8 & 0 & 75 \\
\hline Con alcantarillado & 0 & 33 & 22 & 0 & 0 & 0 \\
\hline Con electricidad & 83 & 83 & 22 & 92 & 95 & 75 \\
\hline Internet, teléfono & 0 & 0 & 0 & 0 & 0 & 0 \\
\hline Vivienda propia & 100 & 67 & 78 & 100 & 95 & 50 \\
\hline
\end{tabular}

Fuente: Elaboración propia con base en los cuestionarios aplicados a jefes de familia. 
En las comunidades Boayaku, Unión de Llandia y Veinticuatro de Mayo, hay 8\%, 20\% y $25 \%$ de analfabetos, respectivamente. La educación primaria es baja, ubicándose entre 23\% y 59\% entre las comunidades, mientras que la secundaria tiene muy bajo porcentaje en todas las comunidades con situaciones críticas en Tzawata, Unión de Llandia y Veinticuatro de Mayo. Solo Flor de Bosque tiene un 11\% de bachilleres e igual número en educación superior completa. Por su parte, Boayaku posee $8 \%$ de graduados universitarios. Las comunidades Wayuri, Flor de Bosque, Boayaku y Veinticuatro de Mayo tienen red de agua en un $67 \%, 22 \%, 8 \%$ y $75 \%$, respectivamente, el resto de las comunidades no cuentan con este servicio. Redes de alcantarillado son patrimonio de Wayuri en 33\% y Flor de Bosque en $22 \%$. Entretanto, el porcentaje de viviendas con electricidad es mayor a 75, exceptuando Flor de Bosque con un 22\%. Todas las viviendas de Tzawata y Boayaku son propias, el resto de las comunidades posee entre 50\% y 95\% de viviendas propias.

Si las comunidades no tienen servicio de educación primaria, tampoco tienen otros servicios de educación en general. Las comunidades están en el área, no han migrado a la ciudad que crece $y$ multiplica sus problemas de falta de abastecimiento. Es necesario potenciar la permanencia de la gente en el sector rural, dotándoles de oportunidades estratégicas de uso de los recursos amazónicos que conocen, para fomentar su crecimiento económico, identidad y estabilidad del patrimonio natural.

BASES PARA ESTRATEGIAS DE DESARROLLO LOCAL EN LAS COMUNIDADES AMAZÓNICAS ECUATORIANAS EN ESTUDIO

En el cuadro 7 se presentan las propuestas de las comunidades para impulsar la sostenibilidad ecológica en relación con la Universidad Estatal Amazónica y las entidades del territorio. 


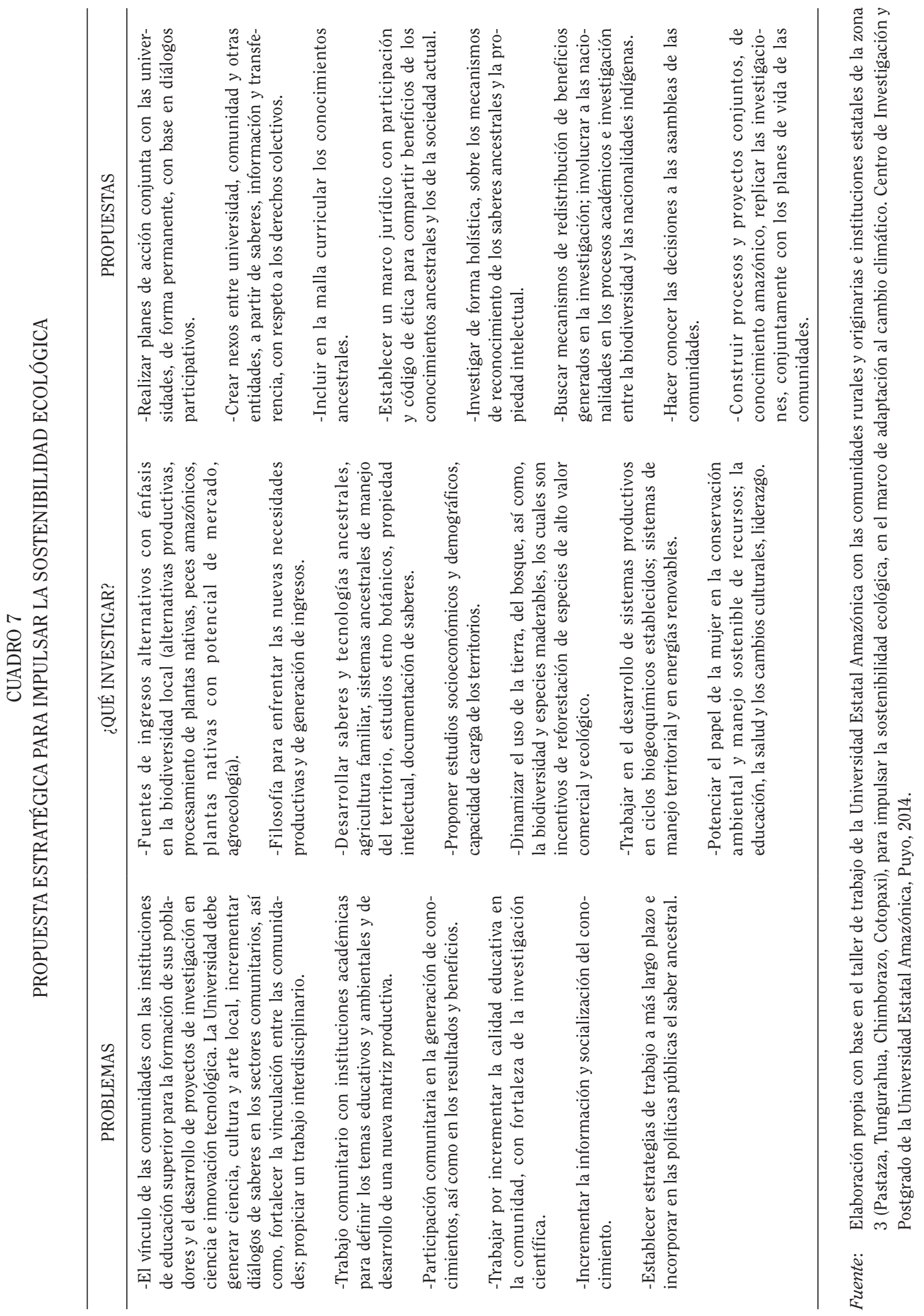

(c) (i) (2) $९$ Rev. Ciencias Sociales Universidad de Costa Rica, 151: 43-57 / 2016 (I). (ISSN: 0482-5276) 
Para enfrentar las necesidades de investigación se propone formar redes de investigación, vinculación, docencia, que incorporen las culturas ancestrales, sus sabios y sus ritos; propiciar una especialización en saberes ancestrales; institucionalizar una política de vinculación con la comunidad en la evaluación académica. Se deben concretar planes de acción con las comunidades en beneficio común.

La estrategia territorial nacional plantea la importancia del bioconocimiento, las redes productivas $y$ de generación tecnológica local y las intervenciones públicas que contribuyan a sostener la ventaja competitiva nacional, basada en la riqueza natural y biológica, con iniciativas estatales universitarias como Yachay, ciudad del conocimiento, e Ikiam, la universidad regional amazónica (Kristiina 2008, Zulay 2008, SenplaDEs 2013). Desde el año 2002, está en funcionamiento la Universidad Estatal Amazónica con sus líneas de investigación y relaciones con las organizaciones representativas de las comunidades del área. Las posibilidades de fomentar una economía popular, social y solidaria abre la oportunidad de interacción entre las comunidades, instituciones del área y la Universidad.

\section{CONCLUSIONES}

Las comunidades indígenas de la Amazonía ecuatoriana mantienen gran cantidad de especies que se conocen por sus usos, sea que se recolecten en el bosque $y$ se regeneren naturalmente o que se cultiven asociadas en sistemas agrícolas; el uso natural no ha desarrollado valor agregado que permita enfrentar los nuevos retos de provisión monetaria para satisfacción de otras necesidades de vestido, movilización, servicios de atención, de ahí que en la interacción entre la universidad, las entidades estatales de la zona de influencia y las comunidades, demande investigar fuentes de ingresos asociadas a la biodiversidad local, a las prácticas y conocimientos desarrollados por la experiencia comunitaria y trasmitidos desde la cultura. Todavía no se ha estudiado con profundidad las especies nativas amazónicas, su manejo, sus beneficios, los arreglos culturales que se vinculan, para establecer beneficios $y$ estrategias de desarrollo local sostenible en el contexto rural amazónico ecuatoriano.

Pese a los procesos de colonización e incursión de otras sociedades en el contexto amazónico, las comunidades locales conservan espacios naturales con mayor riqueza de recursos en relación a las áreas vecinas que han sido degradadas por el mismo proceso colonizador. Este mantenimiento de riqueza de especies trae asociada una cultura diferente de interacción con la naturaleza, los recursos, su aprovechamiento, las formas de conocimiento, por lo que se necesita estudiar la comprensión filosófica, socioeconómica y demográfica de las relaciones múltiples con el entorno, en el marco de interculturalidad, de las nuevas necesidades productivas y de generación de ingresos, como espacios de fronteras internas entre procesos, tiempos, paisajes y relaciones.

Existe capacidad indígena para adaptarse a los cambios drásticos en el medio amazónico $y$ para reorganizarse manteniendo sus funciones, identidad y sistemas de conocimiento, pero es necesario el fortalecimiento del vínculo de las comunidades en estudio con las instituciones de educación superior del territorio, para la formación de sus pobladores y el desarrollo de proyectos de investigación en ciencia e innovación tecnológica. La generación de ciencia, cultura y arte local contribuirá al desarrollo en las comunidades, en un contexto de sostenibilidad.

\section{BIBLIOGRAFÍA}

LIBROS

Cañadas Cruz, L. 1983. El mapa bioclimático y ecológico del Ecuador. MAG-PRONAREG. Quito.

García Linera, Álvaro. 2012. Geopolítica de la Amazonía, poder hacendal-patrimonial y acumulación capitalista. Bolivia: Vicepresidencia del Estado-Presidencia de la Asamblea Legislativa Plurinacional.

González, Roberto y Eduardo Salinas. 2010. Geografía de América Latina. Cuba: Editorial Félix Varela.

Gudynas, Eduardo. 2004. Ecología, economía y ética del desarrollo sostenible. Uruguay: 
Editorial Centro Latino Americano de Ecologia Social-CLAES.

Senplades e Instituto Geográfico Militar-IGm. 2010. Atlas geográfico de la república del Ecuador, población, producción, medio ambiente. Ecuador: Senplades e Instituto Geográfico Militar-IGM.

Senplades. 2013. Plan nacional del buen vivir 2013-2017, estrategia territorial nacional. Ecuador: Senplades.

Sierra, Rodrigo. (Ed). 1999. Propuesta Preliminar de un Sistema de Clasificación de Vegetación para el Ecuador Continental. Ecuador: Instituto Ecuatoriano Forestal y de Áreas Naturales y Vida Silvestre-InEFAn, Fondo para el Medio Ambiente Global- Banco Internacional de Reconstrucción y Fomento GEF-BIRF, $y$ Eco Ciencia.

\section{REVISTAS}

Álvarez, José y Noam Shany. 2012. Una experiencia de gestión participativa de la biodiversidad con comunidades amazónicas. Revista Peruana de Biología 19, 2: 223-232.

Bartolomé, Miguel Alberto. 2010. Interculturalidad y territorialidades confrontadas en América Latina. Runa 31: 9-29.

Gaona Pando, Georgina. 2013. "El derecho a la tierra y protección del medio ambiente por los pueblos indígenas". Nueva antropología 26, 78: 141-161.

Martínez Novo, Rodrigo. 2012. "Del tiempo insostenible y del sentido del tiempo en las comunidades kichwa canelos". Desacatos 40: 111-126.

Montero Mendoza, Elda. 2011. "Percepción de los habitantes indígenas de áreas rurales respecto al primer nivel de atención médica. El caso del sureste de Veracruz, México". Salud colectiva 7, 1: 73-86.

Pérez Garcés Helena y Carlos Alberto Zárate Yepes. 2011. "Turbay Ceballos S. M." Opinión Jurídica, edición especial, 10: 89-104.

Philip, Gondecki. 2011. "Entre retirada forzosa $y$ autoaislamiento voluntario: reflexiones sobre pueblos indígenas aislados y estrategias de evitación en el manejo de conflictos en la Amazonía occidental". Indiana 28: 127-152.

Prates, Luís Enrique. 2009. "Evolución del paisaje amazónico desde el Precámbrico". Revista Brasileira de Geociências 41, 4: 654-661.

Vasco, Cristian; Byron Herrera, Shiram Vargas y Ruth Arias. 2013. "Empleo agrícola y no agrícola en la Amazonía ecuatoriana". Ecuador Debate 90: 141-152.

Vázquez, Héctor. 2012. "Pueblos originarios, cuestión étnico nacional en el cono sur latinoamericano y sus contradicciones con los modelos neodesarrollistas propuestos por los gobiernos progresistas". Pap. trab.-Cent. Estud. Interdiscip. Etnolingüist. Antropol. Sociocult. 23: 98-121.

Zambrana Lara, Carlos. 2011. "Historia fotográfica del puerto de cobija”. Rev. Fuent. Cong. 5, 12: 5-13.

Zulay Poggi González. 2008. "Proyecto de investigación. La protección de los conocimientos tradicionales de las comunidades indígenas de los países Amazónicos. Una propuesta para su preservación". Cuadernos del Cendes 25, 68: 125-127.

\section{TEXTOS ELECTRÓNICOS}

Bilsborrow, Richard, Alisson Barbieri y William Pan. 2004. "Changes in population and land use over time in the Ecuadorian Amazon". Acta Amazonica 34, 4: 635647. Acceso el 12 de marzo de 2013. http://dx.doi.org/10. 1590/S004459672004000400015

Blanke A. y Walzer N. 2013. "Measuring community development: what have we learned?" Community Development 44, 5:534-550. Acceso el 18 de febrero de 2014. http://dx.doi. org/10.1080/15575330.2013. 852595

Cantú Martínez, Pedro César. 2012. "Percepciones sobre medio ambiente. El axioma del desarrollo sustentable". Revista Ciencias Sociales, 137. 
Universidad de Costa Rica: 83-91. Acceso el 4 de diciembre de 2014. http:// www.revistacienciassociales.ucr.ac.cr/ el-axioma-del-desarrollo-sustentable/

Centro de Derechos Económicos y SocialesCDES. 2013. "Plan C: redistribución de la riqueza para no explotar el Yasuní y salvaguardar a los indígenas aislados". Acceso el 13 de setiembre. http://www. observatorio.cdes.org.ec/

Córdoba, Ligia. 1996. "Educación para la no violencia: hacia un desarrollo sostenible, sustentable y sostenido con perspectiva de género". Revista Ciencias Sociales, 71: 123-133. Acceso el 4 de diciembre de 2014. http://www.revistacienciassociales. ucr.ac.cr/educacion-para-la-no-violenciahacia-un-desarrollo-sosteniblesustentable-y-sostenido-con-perspectivade-genero/>

Escobar, Arturo. 2002. "Globalización, desarrollo y modernidad". Planeación, participación y desarrollo. Acceso el 19 de junio de 2014. www.oei.es/salacti/ escobar

Kristiina, Pirjo Virtanen. 2008. "Los procesos de modernización en la Amazonía brasileña. Lógicas sociales de los pueblos indígenas y la interacción entre campos sociales”. Sociológica, 23 67: 51-80. Acceso el 19 de junio de 2015. http:// www.scielo.org. $\mathrm{mx} / \mathrm{scielo}$.php?script $=\mathrm{sci}$ a r t text\&pid=S $0187-017320080$ $00200004 \& \operatorname{lng}=\mathrm{es} \& \mathrm{nrm}=\mathrm{iso}$
OTROS

Instituto Nacional de Estadísticas y Censos de Ecuador-InEc. 2010. Base de datos de resultados del censo de población $y$ vivienda 2010 en el software Redatam. Quito: Instituto Nacional de Estadísticas y Censos de Ecuador-INEC: Censo de Población y de ViviendaCPV 2010-Aplicación de R+SP xPlan. Centro Latinoamericano de Desarrollo Empresarial-Comisión Económica para América Latina y el Caribe (CELADE-CEPAL).

Landázuri, X. 2013. "La visión del Sumak Kawsay desde el pueblo ancestral Kichwa Kuraray-Pinduc". Encuentro por la vida en armonía, el territorio y la multitud de vidas en plenitud de Pastaza. Puyo.

Vacacela, Víctor e Instituto Quichua de Biotecnología Sacha Supai. 2013. "Manejo y conservación de la agro biodiversidad en sistemas de producción ancestral del pueblo Kichwa de Pastaza". Encuentro por la vida en armonía, el territorio y la multitud de vidas en plenitud de Pastaza en la Universidad Estatal Amazónica, Puyo, el 14 y 15 de noviembre.

Fecha de ingreso: 09/12/2014 Fecha de aprobación: 14/12/2015 
